\title{
Bolometric detection of ferromagnetic resonance in YIG slab
}

\author{
Sa Tu, ${ }^{1,2}$ Marcin Białek, ${ }^{2}$ Youguang Zhang, ${ }^{1}$ Weisheng Zhao, ${ }^{1}$ Haiming $\mathrm{Yu},{ }^{1, a)}$ and \\ Jean-Philippe Ansermet ${ }^{2, b}$ ) \\ 1) Fert Beijing Research Institute, School of Electronic and Information Engineering, \\ BDBC, Beihang University, Beijing 100191, Peoples Republic of China \\ ${ }^{2)}$ Institute of Physics, École Polytechnique Fédérale de Lausanne (EPFL), \\ Lausanne, Switzerland.
}

(Dated: April 17, 2017)

The resistance of the $\mathrm{Pt}$ bar deposited on the YIG slab was monitored while the magnetic field was ramped through the ferromagnetic resonance with the YIG slab facing a coplanar waveguide resonator excited at $4.3 \mathrm{GHz}$ excitation. The resistance change provides detection of the ferromagnetic resonance with a high signal-to-noise ratio. It is ascribed to a change in the temperature of the $\mathrm{Pt}$ bars. The thermal origin of the signal is confirmed by the observation that the signal vanishes when field modulation is applied at frequencies above $6 \mathrm{~Hz}$. The spin pumping effect was vanishingly small, and the anisotropic magnetoresistance of the Pt bar, though quite easily observed, would imply a rectification voltage that is much smaller than the bolometric effect.

a) Electronic mail: haiming.yu@buaa.edu.cn

b) Electronic mail: jean-philippe.ansermet@epfl.ch 
Electrical detection of the ferromagnetic resonance (FMR) based on the spin pumping effect $^{1-5}$ has recently drawn great attention in the field of spintronics. The spin current generated by the FMR is injected through an interface between ferromagnetic material (FM) and normal metal (NM) and, due to the inverse spin Hall effect, a DC response can be detected. The spin pumping innately demands high quality interfaces. Yttrium iron garnet (YIG) with a Pt bar deposited on top of it is one commonly used hybrid structures. ${ }^{6-8}$ The experimentally detected electrical voltage signal may have other contributions, most notably the spin rectification effect due to the anisotropic magnetoresistance (AMR) of $\mathrm{Pt}^{9-11}$ and the bolometric effect due to the heat associated with microwave absorption ${ }^{12}$. One may distinguish spin pumping and spin rectification effect based on their different angular dependence ${ }^{13-16}$. When a current is applied either through the sample or through the deposited metal bar, the bolometric effect is another possible contribution to the electrical signal. This has already been shown to be a promising applications in THz technology ${ }^{17,18}$. Gui et al. ${ }^{12}$ in their seminal study show a comparison between spin rectification and bolometric effects in Permalloy. However, very few studies on the bolometric effect in YIG/Pt structures can be found ${ }^{19}$. In this work, we present experimental results showing a distinct bolometric effect as a viable electrical detection of the FMR in YIG/Pt. A voltage response as large as $40 \mu \mathrm{V}$ is observed. It is two orders of magnitude larger than what was reported in previous studies ${ }^{19}$. We will show that the signal becomes very small when the ferromagnetic film is very thin. By varying the magnetic field modulation frequency, we demonstrate that the bolometric effect is the dominant contribution in our observation and that the response of spin rectification and spin pumping is below $1 \mu \mathrm{V}$.

Fig. 1 shows a schematic illustration of our sample and the experimental setup. The sample consists of yttrium iron garnet $(10 \mu \mathrm{m}) / \mathrm{Pt}(10 \mathrm{~nm})$. YIG was grown on a substrate of Gadolinium Gallium Garnet (111) using LPE. Ar ${ }^{+}$plasma cleaning at energies of 50 to $100 \mathrm{eV}$ for 10 min was used to obtain a clean interface before Pt deposition ${ }^{6}$.Afterwards,a Pt strip of $1.5 \times 4 \mathrm{~mm}^{2}$ surface was prepared by sputtering. FMR is excited at frequencies in the range of 4 to $7 \mathrm{GHz}$ using a coplanar waveguide resonator (CPW) and measured via the derivative of microwave power absorption using a small field modulation of 0.3 Oe and lock-in detection. The magnetic field, which is ramped slowly between $-2.5 \mathrm{kOe}$ and $2.5 \mathrm{kOe}$, is applied perpendicular in plane to the Pt strip. All the measurements were performed with a microwave source power of $P_{\mathrm{mw}}=16 \mathrm{dBm}$. In order to measure the resistance in YIG/Pt, 
we used either a nano-voltmeter when there was no field modulation, or a lock-in amplifier when field modulation was applied.

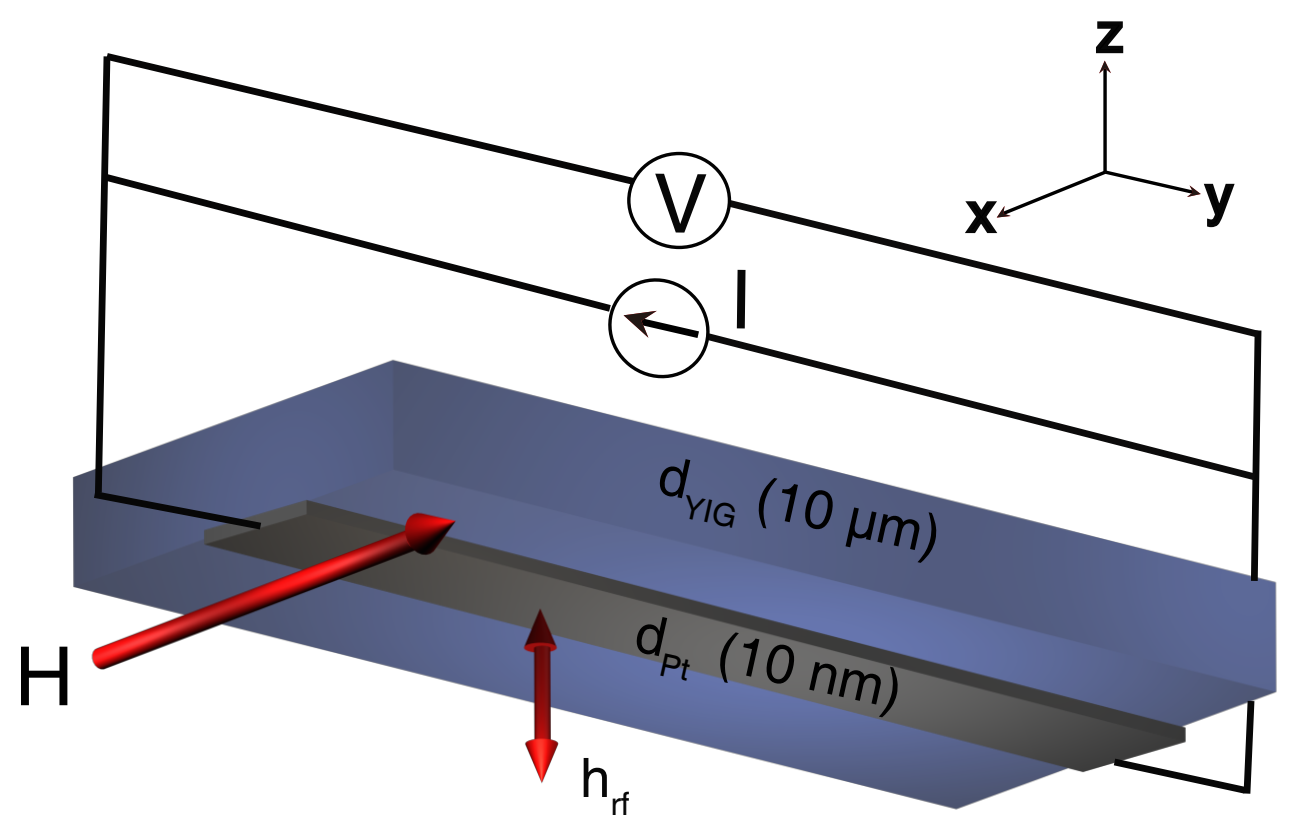

Figure 1. Schematic illustration of the experimental system: FMR signal is excited by CPW.

Our aim was to detect the FMR signal in YIG/Pt electrically. There are three possible mechanisms : the magnetic proximity effect ${ }^{20,21}$, the bolometric effect and the spin pumping. In order to test the first two, which correspond to resistive measurements, we applied a constant current of $100 \mu \mathrm{A}, 1 \mathrm{~mA}$ or $5 \mathrm{~mA}$ to the Pt bars. Considering that the maximum current density of $\mathrm{Pt}$ to avoid damage to be of about $10^{6} \mathrm{~A} / \mathrm{cm}^{2}, 30 \mathrm{~mA}$ would be the upper limit in our case. We find a significant signal at the resonance field when the applied current is less than $100 \mu \mathrm{A}$. An obvious resistance peak appears at 850 Oe (Fig. 2(a)). The signal amplitude can reach as much as $40 \mu \mathrm{V}$ with an applied current of $5 \mathrm{~mA}$ (Fig. 2(b)), which is two orders of magnitude larger than that observed by Hahn et al. ${ }^{19}$ using the same DC current.

We find that the detected signal remains of the same sign when the field is reversed (Fig. 2(a)). Spin pumping voltage response would change sign with a reversed field. ${ }^{22}$ On the contrary, the bolometric effect keeps the same sign at positive and negative field at resonance since it is due to heating resulting from the resonant microwave absorption. 

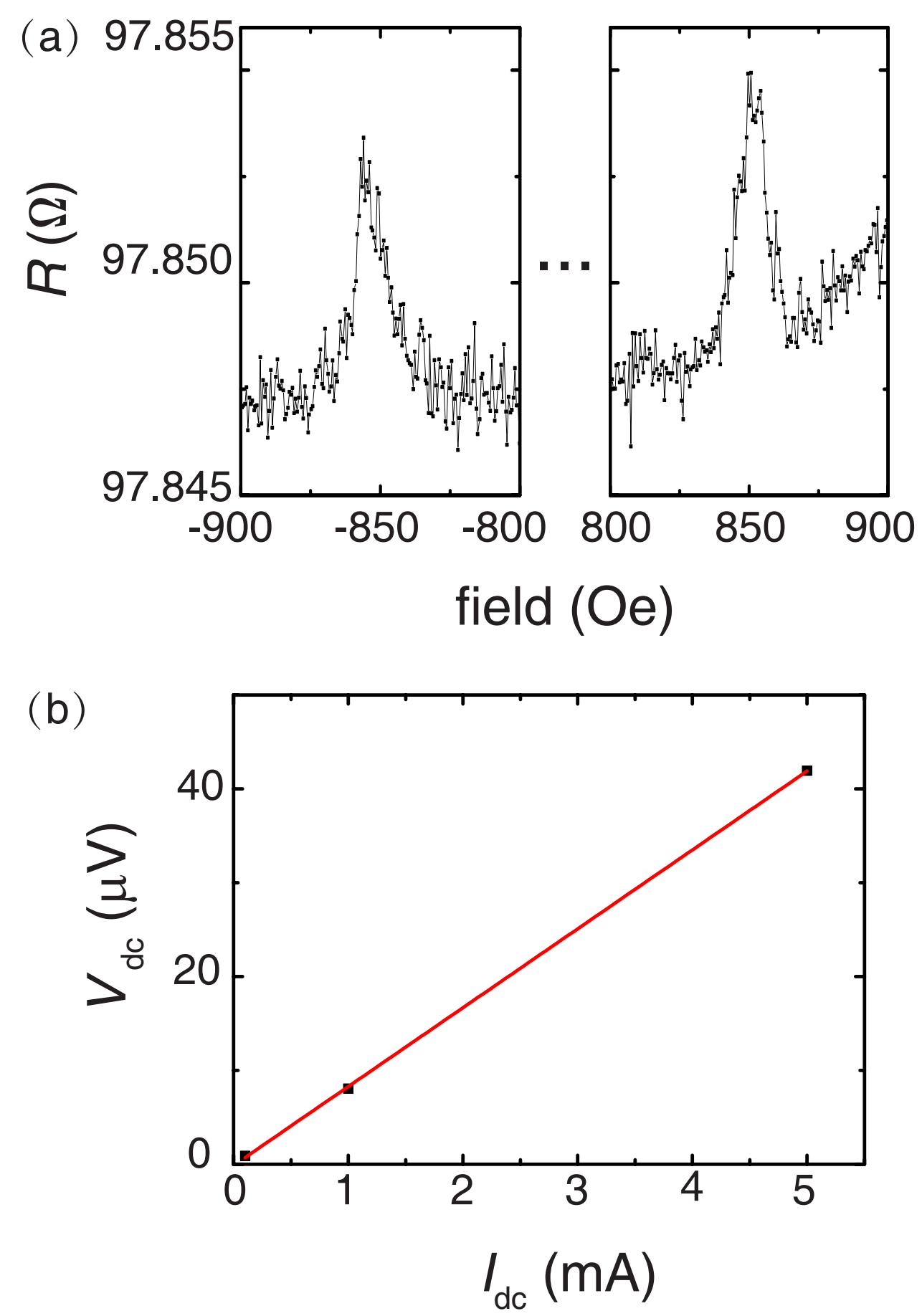

Figure 2. (a) Resistance of YIG/Pt hybrid structure at positive and negative field with $1 \mathrm{~mA}$ current, (b) The magnitude of voltage maximum detected on Pt bar with different applied currents of $100 \mu \mathrm{A}, 1 \mathrm{~mA}$ and $5 \mathrm{~mA}$ at $4.3 \mathrm{GHz}$.

We confirm that this is the resonance position by the regular detection method, moni- 
toring the reflected signal coming back from the resonator (Fig. 3(a)). The resonance field at about 850 Oe for an excitation frequency of $4.3 \mathrm{GHz}$ is what is expected from the Kittel formula ${ }^{23}$ :

$$
\omega=\mu_{0} \gamma \sqrt{H_{0}\left(H_{0}+M_{\mathrm{S}}\right)}
$$

We can distinguish in Fig. 3(a) some small features that correspond to magneto-static modes in this thick YIG slab ${ }^{24}$.

We tried to detect the FMR by monitoring the voltage across the Pt bar in the absence of applied current. That is, we relied on the Inverse spin Hall effect (ISHE) detection of FMR with the Pt strip ${ }^{25}$. Measurement of the ISHE signal was performed either using a lock-in amplifier with a field modulation or a DC nanovoltmeter. No signal was detected using either methods. The most likely reason was that in our sample the surface of the YIG slab was too rough. Because the YIG/Pt interface quality is so critical, most of ISHE experiments are performed on YIG thin film with a thickness in the nm scale.

Fig. 3(b) shows three spectra acquired at three excitation frequencies and detected with a current of $1 \mathrm{~mA}$. This detection scheme yields broader and slightly shiftted lines than the regular detection method. It can be understood as follow, when the YIG is excited by microwave at resonance, it heats up the YIG and Pt, the thermal relaxation could not follow immediately as magnetodynamics leads to line shifting. Since the synthetic signal includes rectification and bolometric signal. It could be possibly become broader combining rectification signal with the same shape and bolometric signal with line shifting. The same observation was made by Goennenwein et $\mathrm{al}^{26}$ and by Hahn et $\mathrm{al}^{19}$.

We can account for the order of magnitude of the effect with a one-dimensional model. We consider that the part of the YIG sample that is subjected to the the highest microwave field just above the hot spot of the CPW resonator heats up because it absorbs power (Fig. 5). We note $P_{Q}$ the power absorbed by unit volume and $C$ the specific heat per unit volume of YIG. Applying the Fourier law $\boldsymbol{j}_{Q}=-\kappa \boldsymbol{\nabla} T$ in the heated column of YIG, we arrive at a modified heat equation that includes the heat source $P_{Q}$ :

$$
C \frac{\partial T}{\partial t}=\kappa \nabla^{2} T+P_{Q}
$$

where $\kappa$ is the heat conductivity. We take the boundary condition that the GGG substrate remains at the ambient temperature $T_{0}$. We assume also that there is no heat dissipation 

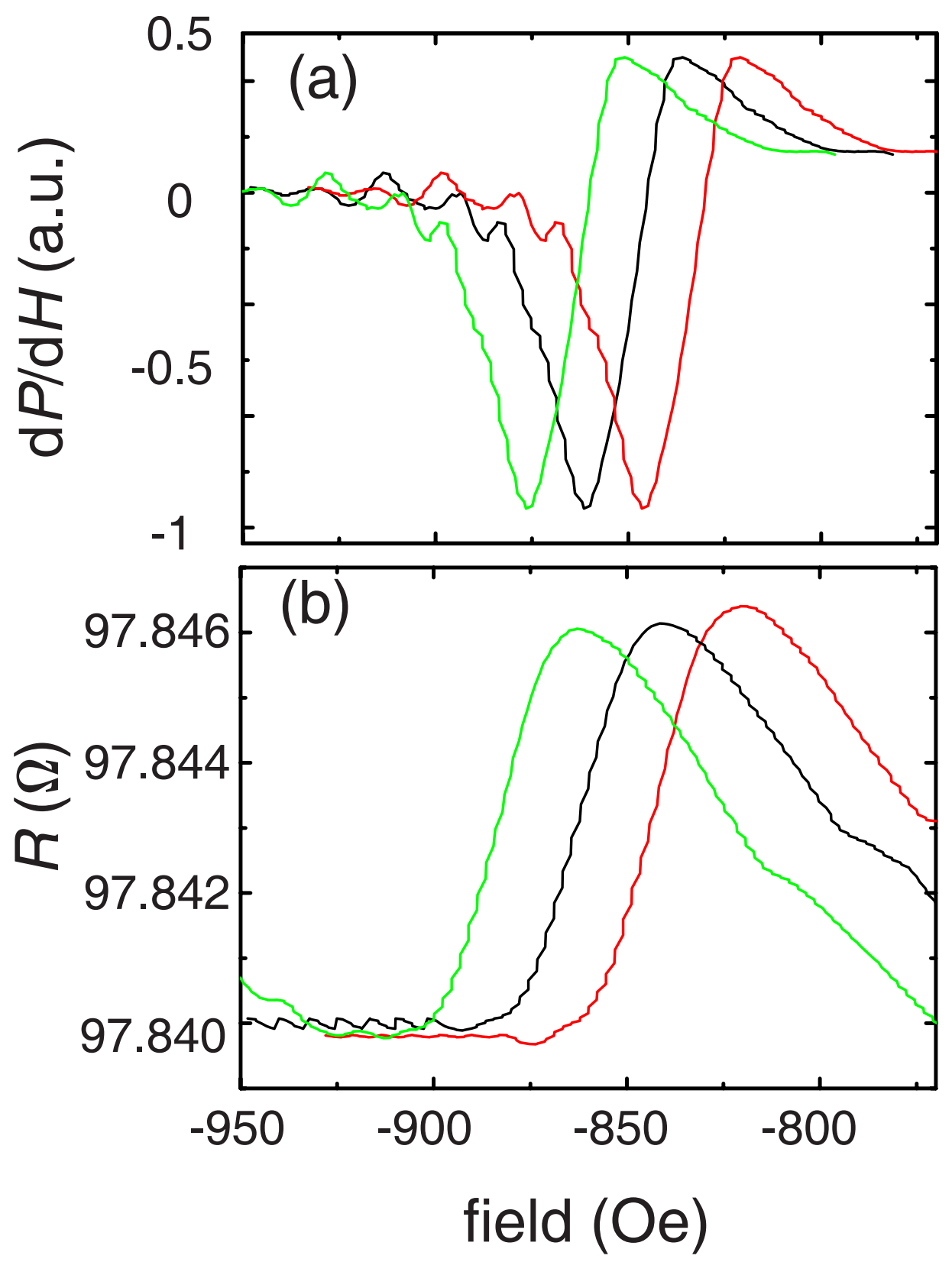

Figure 3. (a) FMR absorption derivative spectra of $10 \mu \mathrm{m}$ thick YIG films at an excitation frequency of $4.2 \mathrm{GHz}$ (red), $4.3 \mathrm{GHz}$ (black) and $4.4 \mathrm{GHz}$ (green). (b) Magnetoresistance measurement at excitation frequencies of $4.2 \mathrm{GHz}$ (red), $4.3 \mathrm{GHz}$ (black) and $4.4 \mathrm{GHz}$ (green) with applied DC current of $1 \mathrm{~mA}$.

at the air interface, $\boldsymbol{j}_{Q}(z=d)=0$, where $d$ is the thickness of the YIG sample. In the 
stationary regime, the integration of $\mathrm{Eq}(2)$ is straightforward, yielding :

$$
T(z)=\frac{-P_{Q}}{2 \kappa} z^{2}+\frac{P_{Q} d}{\kappa} z+T_{0}
$$

Thus, we find that the temperature at the surface of YIG is given by,

$$
T(d)=\frac{P_{Q} d^{2}}{2 \kappa}+T_{0}
$$

We estimate the part of the sample that is irradiated by strong microwave signals to have a surface area $A$ of about $1 \mathrm{~mm}^{2}$ and $d$ in depth. The power $P_{0}$ was $16 \mathrm{dBm}$, and $P_{Q}=\frac{P_{0}}{A d}$. These numerical values imply a temperature rise of $20 \mathrm{mK}$. The Pt bar had a resistance of about $98 \Omega$ and its temperature dependence was about $0.5 \Omega / \mathrm{K}$. Thus, this temperature change provokes a resistance change of $0.01 \Omega$. As the current used to measure the data of Fig 2(a) was of $1 \mathrm{~mA}$, this implies a voltage change upon resonance of $10 \mu \mathrm{V}$.

Therefore, it is resonable to assume that the detected signal arises from the bolometric effect $^{27,28}$ due to the microwave absorption at the resonance, causing a temperature rise of the sample and thereby, changing the resistance of $\mathrm{Pt}$ in close contact to it.

Gui et al. confirmed the thermal origin of their signal by using the power modulation. They estimated the resistance change as $\Delta R_{\mathrm{T}}=\frac{P_{0} \tau}{C_{0}} \frac{d R}{d T}$, where $P_{0}$ is the absorption MW power, $\tau$ is the thermal relaxation time, and $C_{0}$ is the absolute heat capacity of YIG/Pt. ${ }^{12}$

Likewise, we characterize the extent to which a resistance change is of thermal origin, using field modulation and measuring the signal dependence on the modulation frequency $f_{\text {mod }}$. The magnitude of the signal at resonance was measured as a function of $f_{\text {mod }}$ (Fig. 4). If the modulation period is shorter than $\tau$, the thermal relaxation could not follow, which means $\Delta R_{\mathrm{T}}$ is significantly reduced. In other words, $\Delta R_{\mathrm{T}}$ is strongly dependent on $f_{\bmod }$. Fig. 4 demonstrates how thermal relaxation $\tau$ affects the bolometric effect. We deduce from the data the value of $\tau$ to be $0.3 \mathrm{~s}$. Since AMR in our sample was $\frac{\Delta R_{\mathrm{AMR}}}{R}=7 \times 10^{-4}$ which is comparable to the result of Chien et al. ${ }^{20}$, we ascribe the high frequency limit to spin rectification. It cannot be spin pumping, because the signal we observe is proportional to the current (Fig. $2(\mathrm{~b})$ ).

This time constant can be understood as follows. There is one column of YIG, of depth $d$ and surface area $A$ that is heated up (Fig. 5). This heat is communicated to the whole of the YIG sample by a heat current going through a surface of area $S$. This heat is driven by a temperature gradient, which is of the order of magnitude of $\left(T-T_{0}\right) / d$. Thus, as a rough 


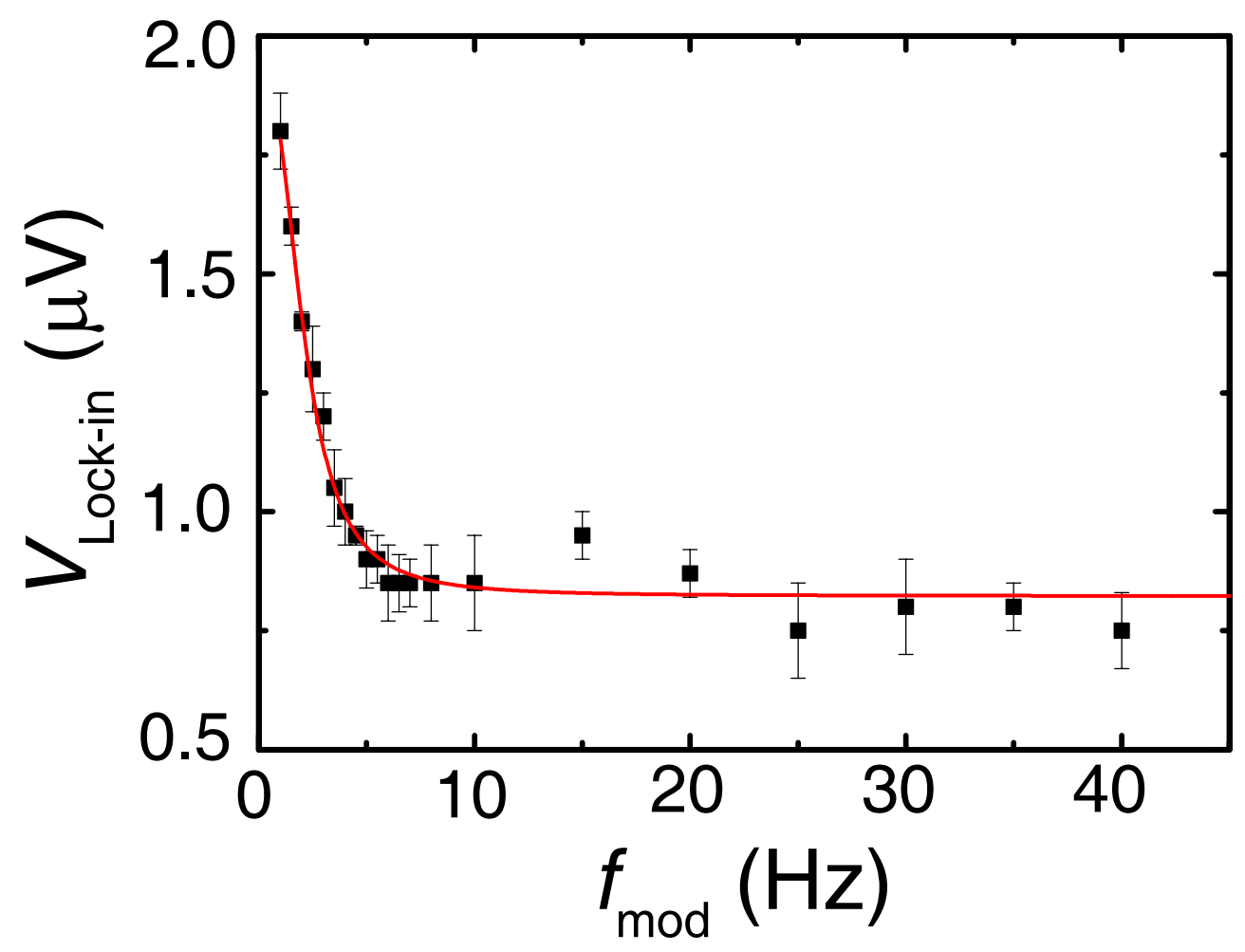

Figure 4. Lock-in detected voltage response as a function of the field modulation frequency up to $40 \mathrm{~Hz}$ with the microwave frequency of $4.3 \mathrm{GHz}$ and the DC current of $1 \mathrm{~mA}$. Error bars indicate noise level at each measured spectrum.

estimate, the dynamics of heating is modeled by :

$$
C V \frac{\partial T}{\partial t}=-\kappa \frac{T-T_{0}}{d} S
$$

which can be rewritten as

$$
\tau \frac{\partial\left(T-T_{0}\right)}{\partial t}=-\left(T-T_{0}\right)
$$

with

$$
\tau=\frac{C}{\kappa} \frac{V d}{S}
$$

Given that $S$ is about $1 \mathrm{~mm}$ times the thickness $d$ of YIG, this rough estimate yieds a time constant $\tau$ of $0.2 \mathrm{~s}$, which is of the right order of magnitude compared to our actual data.

In conclusion, we have studied the resistance change under the microwave irradiation in YIG/Pt. The observed voltage, appearing at the ferromagnetic resonance field, exhibits a dependence on the field modulation frequency which is characteristic of the bolometric 


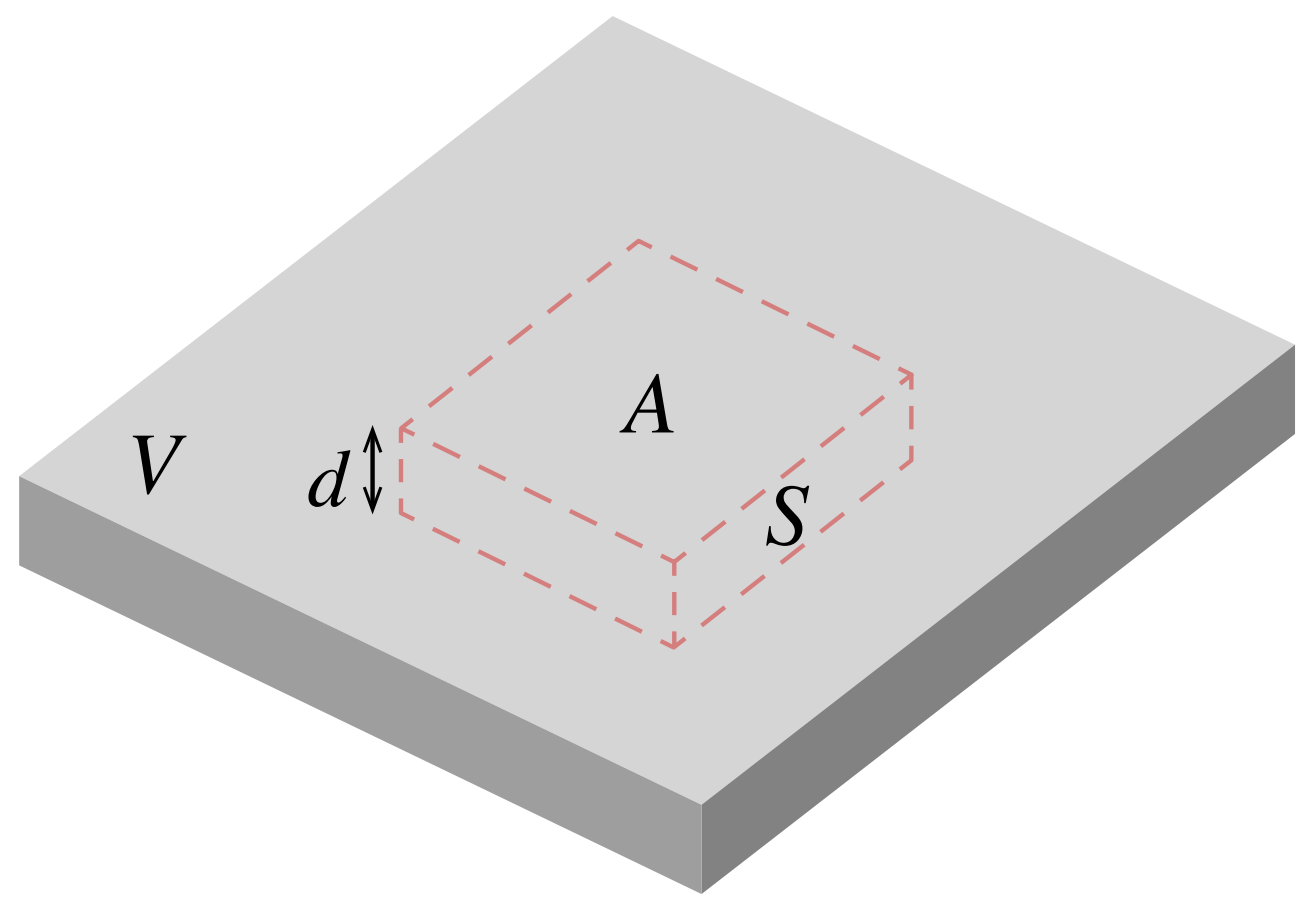

Figure 5. Schematics of the sample (outer frame) and the region (red) of strong microwave irradiation produced by the CPW. Notation : d, thickness of YIG, A, surface area of the region exposed to strong microwave, S, surface area of the section through which heat propagates in YIG, $\mathrm{V}$, volume in which heating occurs.

effect, as it has a time constant characteristic related to heat diffusion in the sample (Eq. 7). The effect scales with the square of the thickness (Eq. 4), hence becomes very small if one were to study FMR in YIG films of only $10 \mathrm{~nm}$ in thickness, as in Ref. 19.

We wish to acknowledge the support by NSF China under Grant No. 11674020 and 11444005, for S.T. by the Sino-Swiss Science and Technology Cooperation SSSTC Grant No.EG 01-032015, for H.Y. by the Deutsche Forschungsgemeinschaft SPP 1538 (SpinCat) grant No. AN762/1, and by the Program of Introducing Talents of Discipline to Universities in China "111 Programme" No. B16001.

\section{REFERENCES}

${ }^{1}$ M. V. Costache, M. Sladkov, S. M. Watts, C. H. van der Wal, and B. J. van Wees. Electrical Detection of Spin Pumping due to the Precessing Magnetization of a Single Ferromagnet. 
Physical Review Letters, 97(21):216603, nov 2006.

${ }^{2}$ B. Heinrich, C. Burrowes, E. Montoya, B. Kardasz, E. Girt, Young-Yeal Song, Yiyan Sun, and Mingzhong Wu. Spin Pumping at the Magnetic Insulator (YIG)/Normal Metal (Au) Interfaces. Physical Review Letters, 107(6):066604, aug 2011.

${ }^{3}$ C. Burrowes, B. Heinrich, B. Kardasz, E. A. Montoya, E. Girt, Yiyan Sun, Young-Yeal Song, and Mingzhong Wu. Enhanced spin pumping at yttrium iron garnet/Au interfaces. Applied Physics Letters, 100(9):092403, feb 2012.

${ }^{4}$ Jung-Chuan Lee, Leng-Wei Huang, Dung-Shing Hung, Tung-Han Chiang, J. C. A. Huang, Jun-Zhi Liang, and Shang-Fan Lee. Inverse spin Hall effect induced by spin pumping into semiconducting ZnO. Applied Physics Letters, 104(5):052401, feb 2014.

${ }^{5}$ S. T. Chui and Z. F. Lin. Large voltage from spin pumping in magnetic tunnel junctions. Physical Review B, 77(9):094432, mar 2008.

${ }^{6}$ M. B. Jungfleisch, V. Lauer, R. Neb, A. V. Chumak, and B. Hillebrands. Improvement of the yttrium iron garnet/platinum interface for spin pumping-based applications. Applied Physics Letters, 103(2):022411, jul 2013.

${ }^{7}$ O. d'Allivy Kelly, A. Anane, R. Bernard, J. Ben Youssef, C. Hahn, A H. Molpeceres, C. Carrétéro, E. Jacquet, C. Deranlot, P. Bortolotti, R. Lebourgeois, J.-C. Mage, G. de Loubens, O. Klein, V. Cros, and A. Fert. Inverse spin Hall effect in nanometerthick yttrium iron garnet/Pt system. Applied Physics Letters, 103(8):082408, aug 2013. ${ }^{8}$ H. Wu, C. H. Wan, X. Zhang, Z. H. Yuan, Q. T. Zhang, J. Y. Qin, H. X. Wei, X. F. Han, and S. Zhang. Observation of magnon-mediated electric current drag at room temperature. Physical Review B, 93(6), feb 2016.

${ }^{9} \mathrm{H}$. J. Juretschke. Electromagnetic Theory of dc Effects in Ferromagnetic Resonance. Journal of Applied Physics, 31(8):1401, 1960.

${ }^{10}$ Y. S. Gui, S. Holland, N. Mecking, and C. M. Hu. Resonances in Ferromagnetic Gratings Detected by Microwave Photoconductivity. Physical Review Letters, 95(5):056807, jul 2005.

${ }^{11}$ Y. S. Gui, N. Mecking, X. Zhou, Gwyn Williams, and C.-M. Hu. Realization of a Room-Temperature Spin Dynamo: The Spin Rectification Effect. Physical Review Letters, 98(10):107602, mar 2007.

${ }^{12}$ Y. S. Gui, N. Mecking, A. Wirthmann, L. H. Bai, and C.-M. Hu. Electrical detection of the ferromagnetic resonance: Spin-rectification versus bolometric effect. Applied Physics 
Letters, 91(8):082503, aug 2007.

${ }^{13}$ O. Mosendz, J. E. Pearson, F. Y. Fradin, G. E. W. Bauer, S. D. Bader, and A. Hoffmann. Quantifying Spin Hall Angles from Spin Pumping: Experiments and Theory. Physical Review Letters, 104(4):046601, jan 2010.

${ }^{14}$ Lihui Bai, Z. Feng, P. Hyde, H. F. Ding, and C.-M. Hu. Distinguishing spin pumping from spin rectification in a $\mathrm{Pt} / \mathrm{Py}$ bilayer through angle dependent line shape analysis. Applied Physics Letters, 102(24):242402, jun 2013.

${ }^{15}$ H. Nakayama, K. Ando, K. Harii, T. Yoshino, R. Takahashi, Y. Kajiwara, K. Uchida, Y. Fujikawa, and E. Saitoh. Geometry dependence on inverse spin Hall effect induced by spin pumping in Ni81Fe19/Pt films. Physical Review B, 85(14):144408, apr 2012.

${ }^{16}$ Dong-Jun Kim, Sang-Il Kim, Seung-Young Park, Kyeong-Dong Lee, and Byong-Guk Park. Ferromagnetic resonance spin pumping in CoFeB with highly resistive non-magnetic electrodes. Current Applied Physics, 14(10):1344-1348, oct 2014.

${ }^{17}$ L. Vicarelli, M. S. Vitiello, D. Coquillat, A. Lombardo, A. C. Ferrari, W. Knap, M. Polini, V. Pellegrini, and A. Tredicucci. Graphene field-effect transistors as room-temperature terahertz detectors. Nature Materials, 11(10):865-871, sep 2012.

${ }^{18}$ A. V. Muraviev, S. L. Rumyantsev, G. Liu, A. A. Balandin, W. Knap, and M. S. Shur. Plasmonic and bolometric terahertz detection by graphene field-effect transistor. Applied Physics Letters, 103(18):181114, oct 2013.

${ }^{19}$ C. Hahn, G. de Loubens, O. Klein, M. Viret, V. V. Naletov, and J. Ben Youssef. Comparative measurements of inverse spin Hall effects and magnetoresistance in YIG/Pt and YIG/Ta. Physical Review B, 87(17):174417, may 2013.

${ }^{20}$ S. Y. Huang, X. Fan, D. Qu, Y. P. Chen, W. G. Wang, J. Wu, T. Y. Chen, J. Q. Xiao, and C. L. Chien. Transport Magnetic Proximity Effects in Platinum. Physical Review Letters, 109(10):107204, sep 2012.

${ }^{21}$ Xiao Liang, Yupeng Zhu, Bo Peng, Longjiang Deng, Jianliang Xie, Haipeng Lu, Mingzhong $\mathrm{Wu}$, and Lei Bi. Influence of Interface Structure on Magnetic Proximity Effect in Pt/Y3Fe5O12Heterostructures. ACS Applied Materials \& Interfaces, 8(12):8175-8183, mar 2016.

${ }^{22}$ Philipp Ross, Michael Schreier, Johannes Lotze, Hans Huebl, Rudolf Gross, and Sebastian T. B. Goennenwein. Antiferromagentic resonance detected by direct current voltages in MnF2/Pt bilayers. Journal of Applied Physics, 118(23):233907, dec 2015. 
${ }^{23}$ Charles Kittel. On the Theory of Ferromagnetic Resonance Absorption. Physical Review, 73(2):155-161, jan 1948.

${ }^{24}$ Elisa Papa, Stewart E. Barnes, and Jean-Philippe Ansermet. Local Excitation of Magnetostatic Modes in YIG. IEEE Transactions on Magnetics, 49(3):1055-1059, mar 2013.

${ }^{25}$ A. V. Chumak, A. A. Serga, M. B. Jungfleisch, R. Neb, D. A. Bozhko, V. S. Tiberkevich, and B. Hillebrands. Direct detection of magnon spin transport by the inverse spin Hall effect. Applied Physics Letters, 100(8):082405, feb 2012.

${ }^{26}$ S. T. B. Goennenwein, S. W. Schink, A. Brandlmaier, A. Boger, M. Opel, R. Gross, R. S. Keizer, T. M. Klapwijk, A. Gupta, H. Huebl, C. Bihler, and M. S. Brandt. Electrically detected ferromagnetic resonance. Applied Physics Letters, 90(16):162507, apr 2007.

${ }^{27}$ Jinwei Rao, Xiaolong Fan, Li Ma, Hengan Zhou, Xiaobing Zhao, Jing Zhao, Fengzhen Zhang, Shiming Zhou, and Desheng Xue. Observation of spin rectification in Pt/yttrium iron garnet bilayer. Journal of Applied Physics, 117(17):17C725, may 2015.

${ }^{28}$ P. Wang, S. W. Jiang, Z. Z. Luan, L. F. Zhou, H. F. Ding, Y. Zhou, X. D. Tao, and D. Wu. Spin rectification induced by spin Hall magnetoresistance at room temperature. Applied Physics Letters, 109(11):112406, sep 2016. 